\title{
Правовые требования к Использованию русского языка в России
}

\section{Сергей Белов}

DOI: 10.30547/mediaalmanah.6.2020.152163

(c) Белов Сергей Александрович кандидат юридических наук, декан юридического факультета СПбГу, директор Научно-исследовательского института проблем государственного языка СПбГу, заведующий кафедрой конституционного права (г. Санкт-Петербург, Россия), s.a.belov@spbu.ru
Правовое регулирование языка, то есть речевого поведения граждан, - вопрос, который лежит на стыке двух предметных областей: правоведения и лингвистики. Цели и общие принципы, пределы и условия правового регулирования, его инструментарий - все это предопределяется логикой права, его социальными функциями. Однако вмешательство в какой-либо социальный предмет требует учитывать его особенности, присущие ему свойства и закономерности. Язык в этом отношении, как предмет правового регулирования, особо сложен, поскольку в нем трудноразличимы две сферы: одна поддается социальному нормированию, другая неподвластна никаким нормам и правилам и составляет область индивидуального речевого творчества, элемент личности, а также инструмент мышления каждого человека. Граница между этими двумя сферами по-разному видится в логике права и в логике языкознания. Для права предметом регулирования может выступать любое речевое поведение, если оно имеет социальное значение, несет в себе риски неблагоприятных социальных последствий разного масштаба. Для лингвистики лишь небольшой круг вопросов использования языка доступен для правового регулирования, поскольку язык функционирует по собственным закономерностям и правилам, сознательное 


\title{
Les normes juridiques relatives à la langue russe en Russie
}

Sergueï Belov

\author{
(C) Belov Sergueï \\ docteur en droit, directeur de la faculté de droit de l'Université d'État de Saint-Pétersbourg, directeur \\ de l'Institut de recherches scientifiques sur les questions de langue officielle de l'État (Université d'État \\ de Saint-Pétersbourg), titulaire de la chaire de droit constitutionnel \\ (Saint-Pétersbourg, Russie), s.a.belov@spbu.ru
}

La réglementation juridique de la langue, c'est-à-dire le comportement langagier des citoyens, est une question qui se situe à la jonction de deux domaines : le droit et la linguistique. Les missions et objectifs, les principes généraux, les limites et conditions de la réglementation juridique, ses outils : tout cela est prédéterminé par la logique du droit, par ses fonctions sociales. Cependant, l'intervention dans un domaine social nécessite de prendre en compte ses particularités, ses propriétés inhérentes et ses régularités. La langue, en tant que sujet de réglementation juridique, est à cet égard particulièrement sensible car il est difficile de distinguer la partie qui est soumise à la réglementation sociale de celle qui se situe au-delà de toute norme et règle et qui constitue le domaine de création de la parole individuelle, élément de la personnalité et instrument de pensée propre à chaque personne. La frontière entre ces deux parties est perçue différemment dans la logique du droit et dans celle de la linguistique. Pour le droit, l'objet de la réglementation peut être n'importe quel comportement discursif s'il a une signification sociale, s'il peut avoir des conséquences sociales négatives à différentes échelles. Pour la linguistique, seul un petit nombre de questions relatives à l'utilisation des langues peut faire l'objet d'une réglementation juridique puisque la langue fonctionne selon ses propres lois et règles, sur lesquelles une influence délibérée et volontaire n'est possible que dans de rares cas exceptionnels, et qui ne sont pas toujours justifiés.

En 2015, l'Université d'État de Saint-Pétersbourg a créé l'Institut de recherche sur les problèmes de langue officielle de l'État. Il regroupe des représentants de différentes spécialités : des linguistes comme des juristes, des sociologues, des psychologues, des spécialistes en gestion et en informatique participent à la mise en œuvre de projets distincts. Les recherches menées dans le cadre de l'Institut de recherche permettent d'identifier un certain nombre de problèmes propres à la réglementation juridique des relations linguistiques en Fédération de Russie, en considérant cette réglementation de manière globale.

La régulation juridique de la langue s'effectue dans deux directions. La première est l'affirmation du statut des différentes langues, en particulier la proclamation d'une langue comme langue officielle de l'État avec la désignation des domaines dans lesquels elle doit être obligatoirement employée. Les langues 
целенаправленное волевое воздействие на которых возможно лишь в редких, исключительных случаях, да и в них не всегда оправданно.

В Санкт-Петербургском государственном университете в 2015 г. был создан Научноисследовательский институт проблем государственного языка, в состав коллектива которого входят представители разных специальностей - и лингвисты, и правоведы; вместе с ними в реализации отдельных проектов участвуют социологи, психологи, специалисты в области менеджмента и компьютерных наук. Исследования, проводимые в рамках НИИ, позволяют сформулировать ряд проблем, которые характерны для правового регулирования языковых отношений в России, рассматривая такое регулирование комплексно.

Правовое регулирование языка осуществляется по двум направлениям. Первое - закрепление статуса разных языков с обозначением сфер его обязательного использования: для республик, входящих в состав Российской Федерации, предусмотрена возможность провозглашения своего языка государственным. Второе - использование, изучение и развитие языков, не имеющих такого статуса, однако сохраняющих свое положение как часть народной культуры, не рассматриваются в качестве обязательного условия единства страны, обеспечения функционирования различных социальных структур - от государства до средств массовой информации. Само по себе наличие разнообразных юридических положений разных языков порождает наличие проблемы соотнесения этих статусов. Например, как совместить изучение всех языков в школе, обеспечить возможность эффективного общения в официальной сфере и получение образования в вузах, где практически повсеместно используется только общегосударственный язык, с задачей сохранения других языков? Как предотвратить изучение одного языка в ущерб освоению другого? Как создать условия для работы государственных органов на двух или более государственных языках (например, опубликование аутентичных текстов на двух государственных языках)? Но главный вопрос - какие сферы общения отнести к тем, где использование государственного языка должно быть обязательным? Обеспечивая единство информационного, коммуникативного пространства в стране, необходимо сохранить свободу языкового творчества и развития языка. Любое правовое решение должно преследовать исключительно легитимные цели, однако и сами эти задачи, и то, какое правовое регулирование им соответствует, не разработаны ни в правовой науке, ни в языкознании.

Ситуация оказывается еще сложнее, если говорить о другом направлении правового регулирования - «обустройстве» языка (используя термин, употребленный французским лингвистом Бернаром Серкильини), то есть об определении языковых норм, обязательныхдля соблюдения при использовании языка как государственного. Эффективно выполнять функцию обеспечения общегосударственной коммуникации язык можеттолько при условии соблюдения нормы наддиалектной, кодифицированной, полифункциональной и стилистически дифференцированной, общей для всех носителей. В зарубежном языкознании это обозначается «стандартным», а в российском - «литературным» вариантом языка, свободным от региональных и социальных диалектов, общепонятным и зафиксированным как «правильный» вариант в академических словарях и справочниках. Очевидной проблемой отсылки в правовых нормах к такому кодифицированному варианту языка для признания его обязательным при использовании как государственного становятся разные представления о норме у юристов и лингвистов. Если в языкознании норма скорее дескриптивна, она прежде всего результат индуктивного обобщения языковой практики и потому 
qui n'ont pas ce statut conservent leur place dans la culture populaire mais leur utilisation, leur étude et leur développement ne sont pas considérés comme une condition nécessaire à l'unité du pays et au fonctionnement des différentes structures sociales, depuis l'État jusqu'aux médias. En Russie, le statut de langue officielle de l'État est partagé avec les langues nationales officielles des républiques constitutives de la Fédération de Russie. L'existence même de différents statuts juridiques selon les langues différentes pose le problème de la corrélation entre ces statuts. Par exemple, comment combiner l'étude de toutes les langues à l'école, comment assurer, d'une part, la possibilité d'une communication efficace dans le domaine officiel et obtenir une formation dans des établissements d'enseignement supérieur où seule la langue officielle de l'État est utilisée presque partout, avec pour mission de préserver les autres langues? Comment éviter l'apprentissage d'une langue au détriment de celui d'une autre, et ainsi de suite ? Ou comment assurer l'activité des autorités publiques dans deux ou plusieurs langues officielles (pour assurer, par exemple, la publication de textes authentiques dans deux langues officielles) ? Mais la question principale est de savoir quels sont les domaines de communication qui doivent être inclus lorsque l'utilisation de la langue nationale est obligatoire, de préserver la liberté de la créativité linguistique et le développement des langues tout en assurant l'unité de l'espace d'information et de communication dans le pays. Toute solution juridique doit poursuivre exclusivement des objectifs légitimes, mais aussi les objectifs légitimes eux-mêmes et la réglementation juridique correspondant à ces objectifs, ce sont des questions qui ne sont pas formulées dans la science juridique car elles nécessitent l'intervention d'experts en communication, ou en linguistique, dans la mesure où la plupart des linguistes considèrent qu'il s'agit de questions et de mission étrangères qui ne leur reviennent pas mais qui concernent les juristes.

La situation se complique encore si l'on parle d'un autre domaine de la réglementation juridique, à savoir l'« aménagement linguistique » (pour reprendre l'expression utilisée par Bernard Cerquiglini), c'est-à-dire la définition des normes linguistiques dont le respect est obligatoire pour l'emploi d'une langue en tant que langue officielle. Une langue ne peut remplir efficacement la fonction de communication nationale que si sont respectées les normes de langue supradialectale, codifiée, multifonctionnelle et stylistiquement différenciée, commune à tous les locuteurs, les formes d'existence de la langue - ce qui en linguistique est désigné à l'étranger comme " langue standard » et en russe comme variante " littéraire ", exempte de dialectes régionaux et sociaux, généralement comprise et fixée comme variante " correcte » dans les dictionnaires académiques et les ouvrages de référence. Le problème évident de la référence dans les normes juridiques à une telle variante codifiée de la langue pour qu'elle soit reconnue comme obligatoire lorsqu'elle est utilisée comme langue officielle est la variété des avis sur la norme entre les juristes et les linguistes. Si, en linguistique, la norme a plus de chances d'être descriptive, elle est avant tout le résultat d'une généralisation inductive de la pratique linguistique et donc mobile et variable, en droit la norme est une prescription fixe, dont le respect est obligatoire. Bien que juridiquement fondée, l'idée d'utiliser des dictionnaires et des livres de référence de grammaire comme sources de normes à caractère prescriptif soulève 
подвижна и вариативна, то в праве норма это зафиксированное предписание, обязательное для соблюдения. Идея использования словарей и грамматических справочников как источников норм прескриптивных, при этом юридически значимых, вызывает особенно активные возражения со стороны представителей лингвистики, несмотря на то что сама идея нормативного словаря языкознанию известна. Однако вмешательство в тонкую и деликатную материю языка со стороны права, воспринимаемое как нечто чуждое, грубое и посягающее на свободу речи, лингвистами рассматривается как неприемлемое в принципе, и в этом отношении непонимание между языкознанием и юриспруденцией носит наиболее принципиальный характер, что не может не отражаться на реальной практике правового регулирования языка в российском законодательстве.

Уровень языкового разнообразия в России один из самых высоких в мире. По разным подсчетам, к языкам народов России относится от 150 до 200 языков самых разных языковых групп. Правовое закрепление статуса какого-либо языка как государственного многие годы не считалось приемлемым. В частности, такова была политика в СССР, где руководящим принципом был запрет на юридическое вмешательство в языковую свободу, сформулированный еще В. И. Лениным в 1914 г. Признание русского языка государственным в нашей стране произошло незадолго до распада СССР с принятием в октябре 1991 г. Закона РФ «О языках народов РФ». Этот закон носил в целом рамочный характер, содержал много гарантий сохранения, изучения и развития всех языков, но при этом включал мало императивных норм, требовавших обязательного использования государственного языка в каких-либо определенных сферах, например в судопроизводстве (чего требовала еще Конституция СССР 1977 г. для «языков союзных республик», в РСФСР для русского языка, как конкретизировала
Конституция РСФСР 1978 г.). При этом шла работа органов государственной власти публикация законов и других нормативных актов, проведение выборов и референдумов (с оговорками об использовании в республиках РФ наряду с общегосударственным языком государственных языков этих республик). Однако в отношении официального делопроизводства, средств массовой информации, общения граждан с государственными органами регулирование носило диспозитивный характер, т.е. наряду с государственным языком Российской Федерации могли использоваться другие языки народов России, особенно в местах компактного проживания этих народов. В сфере образования гарантировалось право выбора языка обучения, в том числе получения образования на родном языке.

Статус русского как государственного языка Российской Федерации был подтвержден в Конституции, принятой в декабре 1993 г., однако существенные изменения в правовом регулировании произошли только с принятием в 2005 г. специального Федерального закона «О государственном языке РФ», который существенно изменил принципы правового регулирования. Вопервых, в законе гораздо жестче и определеннее были сформулированы требования к обязательному использованию государственного языка в ряде сфер публичного официального общения, круг которых расширился и дополнился за счет обязательного использования в деятельности организаций всех форм собственности, в средствах массовой информации и в рекламе, при публичном показе художественных постановок и фильмов. Во-вторых, закон установил требования не только к статусу языка, но и к вопросам его «обустройства», урегулировав обязательность соблюдения норм современного литературного языка, порядок фиксации которых должно было определить Правительство РФ. Этот порядок был установлен в 2007 г., когда Правительство передало Министерству 
des objections particulièrement fortes de la part des représentants de la linguistique, même si l'idée d'un vocabulaire normatif est reconnue des linguistes. Cependant, les linguistes considèrent que l'ingérence de la part de la loi dans la question complexe et délicate de la langue, perçue comme quelque chose d'étranger, de grossier et portant atteinte à la liberté d'expression, est inacceptable par principe, et à cet égard le malentendu entre la linguistique et le droit est fondamental, ce qui ne peut qu'affecter la pratique réelle de la réglementation juridique de la langue dans la législation russe.

La Russie est l'un des pays les plus riches du monde en matière de diversité linguistique. Selon diverses estimations, entre 150 et 200 langues de différents groupes linguistiques font partie des langues des peuples de Russie. Conférer un statut juridique à une langue quelconque en tant que langue officielle a été considéré comme inacceptable pendant de nombreuses années. C'était notamment la politique en URSS, où le principe était d'interdire tout ingérence législative dans la liberté linguistique formulée par Lénine en 1914. La reconnaissance de la langue russe comme langue officielle en Russie s'est faite peu avant l'effondrement de l'URSS, en octobre 199, avec l'adoption de la loi fédérale " Sur les langues des peuples de la Fédération de Russie ». Il s'agissait d'une loi-cadre qui comprenait de nombreuses garanties pour la préservation, l'étude et le développement de toutes les langues et qui, inversement, comportait peu de normes impératives sur l'obligation de l'emploi de la langue officielle des domaines définis. Outre l'emploi obligatoire de la langue officielle dans les procédures judiciaires (ce qui était requis par la Constitution de l'URSS de 1977 pour les « langues des républiques de l'Union ", avec le russe en RSFSR, comme spécifié par la Constitution de la RSFSR de 1978), c'était le travail des autorités de l'État, la publication des lois et autres actes normatifs, la tenue d'élections et de référendums (avec des réserves concernant l'utilisation des langues officielles de ces républiques parallèlement à la langue nationale dans les républiques de la Fédération de Russie). Toutefois, en ce qui concerne les processus administratifs, les médias et la communication entre les citoyens et les organismes d'État, la réglementation revêtait un caractère aléatoire, c'est-à-dire que les autres langues des peuples de Russie pouvaient être utilisées en même temps que la langue officielle de la Fédération de Russie, en particulier dans les lieux où les autres peuples vivent de manière compacte. Le droit de choisir la langue d'enseignement est garanti, y compris le droit de bénéficier d'un enseignement dispensé dans sa langue maternelle.

Le statut du russe en tant que langue officielle de la Fédération de Russie a été confirmé dans la Constitution adoptée en décembre 1993, mais ce n'est qu'avec l'adoption en 2005 de la loi fédérale spéciale sur la langue officielle de la Fédération de Russie, qui a modifié de manière significative les principes de la réglementation juridique, que des changements importants dans ce domaine sont intervenus. Tout d'abord, la loi de 2005 a formulé des normes beaucoup plus strictes et précises concernant l'utilisation obligatoire de la langue de l'État dans un certain nombre de domaines de la communication publique officielle, dont la portée a été élargie et complétée par l'utilisation obligatoire dans les activités des organisations de toutes formes de propriété, dans les médias et dans la publicité et dans la projection publique de longs métrages et de films. Deuxièmement, la loi de 2005 a établi des normes relatives non seulement au 
образования и науки полномочия утверждать перечень словарей и справочников, содержащих нормы современного русского литературного языка, следование которым обязательно при использовании языка в качестве государственного, т.е. в тех сферах, где этого требует закон.

К сожалению, правовое регулирование, которое сформировалось в законе 2005 г. и на его основе, не лишено ряда недостатков. В ряде сфер требование об использовании государственного языка по-прежнему сохраняет усмотрение относительно выбора языка. Одна из наиболее важных сфер - это средства массовой информации. Если Закон о государственном языке требует использования именно русского языка, то в положениях Закона о СМИ сохраняется право учредителя выпускать СМИ на любом другом языке. Такое регулирование не только неопределенно, поскольку состоит из разных правил, имеющих одинаковую юридическую силу, но и не учитывает общественных реалий - потребности выпуска СМИ на разных языках, в том числе на языках коренных народов России. Некоторые сферы (образование, защита прав потребителей, транспорт) в Законе о государственном языке вообще не упоминаются (скрываются за отсылкой к «другим законам»), однако содержащееся в других документах регулирование часто оказывается несогласованным с нормами данного закона.

Один из наиболее сложных и болезненных вопросов правового регулирования и в целом языковой политики в России - это обеспечение получения образования на государственном языке в национальных республиках. Сложности возникают с необходимостью совмещения в рамках школьной программы и общегосударственного языка, и государственного языка республик, и родных языков из числа языков народов России. Поиск баланса между необходимостью изучения государственного языка и правом родителей определять язык обучения для своих детей остается предметом активных общественных дискуссий в национальных республиках, особенно после 2018 г., когда Государственная Дума в третьем, окончательном чтении приняла законопроект о родных языках (основным принципом которого стала свобода в выборе родителями языка, изучаемого ребенком в школе в качестве родного), сохранив обязательность изучения только государственного языка Российской Федерации.

В части «обустройства» русского языка Федеральный закон о государственном языке ввел прямой запрет на употребление иностранных слов, имеющих аналоги в русском языке, а также на нецензурную брань. Ни первый, ни второй запреты не носят достаточно ясного и определенного характера. Утверждать, что заимствуемое слово имеет аналог в русском языке, заведомо бессмысленно, поскольку любое слово имеет определенные оттенки значений, контекст употребления (в том числе принадлежность к какому-то стилю или регистру языка) и тем самым никогда не будет полным аналогом другого слова. Что касается нецензурной брани, то считать это слово (словосочетание) термином, имеющим четкое лингвистическое значение, сложно, словарей с исчерпывающим перечнем таких слов нет, следовательно, это запрещение носит расплывчатый характер. Усугубляется это положение тем, что законодательство о рекламе похожий запрет формулирует иначе, требуя воздерживаться от использования в рекламных материалах «бранных и оскорбительных» слов, в отношении которых неясно, должны ли они обязательно относиться к нецензурной брани, либо этот запрет существует наряду с запрещением, установленным Законом о государственном языке.

Неопределенность любых юридических норм - это само по себе сложное явление, также возникающее на стыке вопросов права и лингвистики. Конституционные - базовые - 
statut de la langue, mais aussi aux questions d'u aménagement linguistique ", réglementant le respect obligatoire des normes de la langue littéraire moderne, la procédure de détermination devant être assumée par le gouvernement de la Fédération de Russie. Cette procédure a été établie en 2007 lorsque le gouvernement a délégué au ministère de l'Enseignement et de la Science le pouvoir d'approuver une liste de dictionnaires, d'ouvrages de référence et de grammaires contenant les normes de la langue littéraire russe moderne qui doivent être suivies dans le cadre de l'emploi de la langue en tant que langue officielle, c'est-à-dire dans les domaines où la loi l'exige.

Malheureusement, la réglementation juridique issue de la loi de 2005 et fondée sur celle-ci n'est pas sans présenter un certain nombre de lacunes.

Dans un certain nombre de domaines, l'obligation d'utiliser la langue officielle reste aléatoire, c'est-à-dire qu'elle reste à la discrétion du choix de la langue. L'un des domaines les plus importants est celui des médias. Alors que la loi sur la langue officielle impose l'utilisation du russe, les dispositions de la loi sur les médias conservent le droit du fondateur d'un média de publier dans toute autre langue. Non seulement cette réglementation est vague, car elle consiste en différentes règles ayant la même force juridique, mais elle ne tient pas compte des réalités sociales : la nécessité de diffuser dans différentes langues, y compris celles des peuples indigènes de Russie. Certains domaines (enseignement, protection des consommateurs, transports) ne sont pas du tout mentionnés dans la loi sur la langue officielle, se cachant derrière une référence à " d'autres lois ", mais la réglementation contenue dans les autres lois est souvent incompatible avec ces normes. L'une des questions les plus complexes et les plus sensibles de la réglementation juridique et de la politique linguistique en Russie en général est l'accès à un enseignement dispensé dans la langue officielle dans les républiques nationales. Les difficultés proviennent de la nécessité de combiner, dans le cadre des programmes scolaires, et langue nationale et langue officielle des républiques et langues maternelles des peuples de Russie. La recherche d'un équilibre entre la nécessité d'étudier la langue officielle et le droit des parents de déterminer la langue d'enseignement de leurs enfants demeure l'objet d'un débat public poussé dans les républiques nationales, surtout depuis 2018 lorsque les amendements à la loi fédérale sur l'enseignement ont proclamé la liberté des parents de choisir la langue à étudier comme langue maternelle tout en conservant l'obligation de n'étudier que la langue officielle de la Fédération de Russie.

En ce qui concerne l'« aménagement » de la langue russe, la loi fédérale sur la langue officielle a introduit l'interdiction directe d'utiliser des mots étrangers ayant des analogues en langue russe et celle du lexique grossier. Ni la première ni la deuxième interdiction ne sont suffisamment claires et précises. Affirmer qu'un mot emprunté possède un analogue en russe est évidemment insensé dans la mesure où tout mot a certaines nuances de sens, de contexte d'utilisation (y compris l'appartenance à un certain style ou registre de langue) et ne sera donc jamais l'analogue complet d'un autre mot. En ce qui concerne le langage grossier, il est difficile de considérer cette combinaison de mots comme un terme ayant une signification linguistique claire, il n'existe pas de dictionnaires donnant une liste exhaustive de ces mots et cette interdiction est donc assez floue. Cette disposition est aggravée par le fait que la législation sur la publicité formule d'une 
принципы права требуют, чтобы все правовые предписания были ясны и определенны. В этом проявляется одна из главных ценностей права - предсказуемость последствий любого поведения. Каждый человек должен отдавать себе отчет в том, какое поведение правом осуждается, к какому право относится нейтрально; за нарушение каких норм наступит ответственность и насколько суровая, каковы могут быть другие последствия несоблюдения правовых норм. Подобную предсказуемость можно обеспечить, только если текстуальная формулировка положения закона не будет содержать очевидных двусмысленностей ни в словах, ни в синтаксических конструкциях. Фактически конституционные принципы предъявляют требования к определенному языку в нормативных правовых актах, тем самым тоже регламентируя использование языка, хотя эти предписания не конкретизируются в законах ни с точки зрения статуса языка, ни сточки зрения его "обустройства».

Пример с особыми требованиями к языку нормативных актов вскрывает еще одну проблему правового регулирования языка необходимость дифференциации предписаний о правилах употребления языка в разных сферах его обязательного использования. К языку нормативных актов претензии даже в пределах соблюдения норм литературного языка явно должны быть не такими же, как к языку рекламы. Общее правило следовать языковой норме охватывает различия в функциональных стилях только при определенном истолковании самого понятия «нормы»: в случае с языком официальных документов норма оказывается более строгой и гораздо менее гибкой, чем в языке рекламы или СМИ. Подобных различий напрямую российский закон не содержит, формально предъявляя одинаковые требования и к языку судопроизводства, и к языку, на котором публично демонстрируются художественные произведения.
Однако этой неясностью неопределенность требований, установленных Законом о государственном языке, не исчерпывается. Описанный выше механизм официальной фиксации языковых норм для целей использования языка в качестве государственного связан с рядом проблем в его реализации на практике. Министерство образования и науки в 2009 г. определило перечень словарей, содержащих нормы современного русского литературного языка при его использовании в качестве государственного, не сформулировав ни требования к таким словарям, ни критерии их отбора. В результате мало кто из лингвистов остался в стороне от критики сделанного министерством выбора. В частности, в перечень попал грамматический словарь А.А. Зализняка - одного из самых авторитетных российских лингвистов. Однако этот словарь не только по организации материала вызывает трудности в использовании даже у некоторых специалистов и совершенно недоступен для обычных граждан, но и явно не носит нормативного характера, определяя не только реально используемые, но и потенциально возможные грамматические формы словообразования. Сложно отнести к нормативным по содержанию и фразеологический словарь под ред. В.Н. Телия. В то же время в перечне словарей отсутствует самый востребованный словарь - толковый. Возможно, с точки зрения лингвиста, в части выявления языковой нормы фиксация значений слов наиболее трудна, однако именно к таким словарям чаще всего прибегают в спорах о языке суды и другие государственные органы, что хорошо видно при анализе правоприменительной практики.

В отличие от ситуации во Франции, в России не пошли по пути выделения наиболее авторитетного научного учреждения, результаты работы которого по кодификации языковой нормы можно считать официальными. Среди специалистов обсуждались несколько институтов, претендующих 
manière différente une interdiction similaire, exigeant que les supports publicitaires s'abstiennent d'utiliser " des jurons et des insultes ", dont on ne sait pas s'ils doivent nécessairement relever du lexique grossier ou si l'interdiction existe parallèlement à celle qui est établie par la loi sur la langue officielle.

L'indétermination des normes juridiques, et pas seulement des normes de la législation sur la langue officielle, est un phénomène complexe en soi qui survient également à la jonction entre les questions juridiques et linguistiques. Les principes juridiques constitutionnels - de base - exigent que toutes les prescriptions juridiques soient claires et définies. C'est là que se manifeste une des principales valeurs du droit : la prévisibilité des conséquences de tout comportement. Cette prévisibilité ne peut être obtenue que si la formulation textuelle d'une disposition de loi n'est pas manifestement ambiguë, que ce soit dans les mots ou dans les constructions syntaxiques. En fait, les principes constitutionnels imposent des normes à l'égard d'une certaine langue dans les actes juridiques, réglementant ainsi également l'utilisation de la langue, bien que ces prescriptions ne soient pas spécifiées dans les lois, ni en termes de statut de la langue ni en termes d'« aménagement ».

L'exemple des exigences particulières pour la langue des actes normatifs révèle un autre problème de la réglementation juridique de la langue, à savoir la nécessité de différencier les prescriptions sur les règles d'emploi de la langue dans les différents domaines de son utilisation obligatoire. La loi russe ne stipule pas directement de telles distinctions, imposant formellement les mêmes exigences quant à la langue de la procédure judiciaire et à celle dans laquelle les œuvres artistiques sont exposées publiquement.

Cependant, l'indétermination des normes énoncées dans la loi sur la langue officielle ne se limite pas à cette ambiguïté. En 2009, le ministère de l'Enseignement et de la Science a établi la liste des dictionnaires contenant les normes de la langue littéraire russe moderne lorsqu'elle est utilisée comme langue officielle, sans formuler ni normes pour ces dictionnaires ni critères pour leur sélection. De ce fait, peu de linguistes se sont abstenus de critiquer le choix fait par le ministère. La liste comprenait notamment le dictionnaire de grammaire d'Andreï Zalizniak, certainement l'un des linguistes russes qui font le plus autorité. Cependant, non seulement ce dictionnaire est difficile à utiliser en raison de son agencement, même pour certains spécialistes, et il est totalement inaccessible aux citoyens ordinaires, mais il n'a manifestement aucun caractère normatif, déterminant non seulement les formes grammaticales de formation des mots réellement utilisées, mais aussi celles qui sont potentiellement possibles. Le dictionnaire phraséologique édité par Véronika Telia est également difficile à classer comme normatif par son contenu. En même temps, il manque à la liste des dictionnaires le dictionnaire le plus populaire, c'est-à-dire explicatif. Du point de vue du linguiste, pour identifier la norme linguistique il est probablement plus difficile de fixer le sens des mots, mais ces dictionnaires sont le plus souvent utilisés dans les litiges concernant la langue par les tribunaux et autres organismes gouvernementaux, ce qui est visible dans l'analyse de la pratique du respect de la loi.

Contrairement à la France, la Russie n'a pas suivi la voie consistant à désigner l'institution scientifique la plus autorisée dont les résultats des travaux en matière de codification de la norme linguistique pourraient être considérés comme 
на роль такого учреждения, - и вся Российская академия наук, и отдельные ее институты, однако Министерство образования и науки пошло по пути включения в перечень словарей конкретных авторов и даже конкретных изданий). Даже за 10 лет, прошедшие с утверждения официального перечня нормативных словарей, языковая норма изменилась, каждый из словарей выдержал уже несколько переизданий, однако формально для признания их официального статуса требуется внесение изменений в приказ Минобрнауки.

Нет в настоящее время определенности и с правилами русской орфографии и пунктуации. Последнее официальное утверждение свода таких правил произошло в 1956 г., а все последующие изменения носили неофициальный характер. Следование этим правилам в настоящее время затруднительно по тем же причинам, что и следование нормам, зафиксированным в официально утвержденных словарях, только изменение языка в случае с правилами орфографии и пунктуации уже исчисляется не годами, а десятилетиями. В связи с этим часто приходится слышать, что эти правила безнадежно устарели и соблюдать их не нужно; формального признания такого устаревания до сих пор нет, и с точки зрения буквы закона следование правилам 1956 г. обязательно.

Наконец, правовое регулирование языка в настоящее время никак не затрагивает одну из наиболее важных социальных проблем - понятности для граждан языка официальных (юридических) документов. В России, как и во многих других странах, юридический язык, который лингвисты часто отказываются признавать профессиональным жаргоном, настаивая на том, что это лишь функциональный стиль литературного языка, за последнее десятилетие значительно усложнился, особенно в части используемых синтаксических конструкций. В результате даже профессиональные юристы начинают испытывать затруднение в понимании текстов законов, подзаконных актов и некоторых судебных решений. Лингвисты уже начали работу по выработке рецептов борьбы с чрезмерным усложнением юридического языка (один из таких проектов реализуется в НИИ проблем государственного языка СПбГУ при поддержке Российского научного фонда), однако для успешного применения этих рекомендаций на практике необходимо признание в правовом регулировании, как минимум, главного принципа - понятности любых юридических текстов тем гражданам, кому они адресованы. В случае с законами это должен быть широкий кругтех, чьи права и обязанности они затрагивают и кого могут привлечь к ответственности за их несоблюдение. Подобные требования, к сожалению, сегодня признаются далеко не всеми правоведами, некоторые настаивают на том, что правовые документы должны быть понятны только специалистам. Однако принципы демократического государства не позволяют считать такой подход приемлемым. В связи с этим одно из главных требований к развитию системы правового регулирования языка в России - это закрепление на уровне правового принципа обязательного стремления к общей понятности любых нормативных правовых актов. 
officiels. Les spécialistes ont débattu de plusieurs institutions pouvant prétendre au rôle d'une telle institution - toute l'Académie des sciences de Russie, certains de ses instituts - mais le ministère a fait le choix d'inscrire dans une liste des dictionnaires spécifiques, en indiquant non seulement les auteurs de ces dictionnaires, mais aussi leurs données de références, c'est-à-dire à une édition bien précise. Même durant les 10 années qui se sont écoulées depuis l'approbation de la liste officielle des dictionnaires normatifs, la norme linguistique a changé, chacun des dictionnaires a connu plusieurs rééditions, mais l'arrêté du ministère de l'Enseignement et de la Science doit être modifié pour que celles-ci puissent être formellement reconnues comme officielles.

À l'heure actuelle, il n'y a pas non plus de définition claire quant aux règles d'orthographe et de ponctuation russes. La dernière approbation officielle d'un ensemble de règles de ce type remonte à 1956, et tous les changements survenus ultérieurement ont été informels. À l'heure actuelle, il est difficile de suivre ces règles, pour les mêmes raisons que suivre les règles enregistrées dans les dictionnaires agréés, sauf que l'évolution de la langue dans le cas des règles d'orthographe et de ponctuation ne se compte plus en années, mais en décennies. Par conséquent, on entend souvent dire que ces règles sont irrévocablement dépassées et n'ont pas besoin d'être suivies ; pourtant, il n'y a toujours pas de reconnaissance formelle de cette obsolescence et, du point de vue de la lettre de la loi, l'adhésion aux règles de 1956 est obligatoire.

Enfin, la réglementation juridique de la langue ignore actuellement l'un des problèmes sociaux les plus importants : celui de la compréhensibilité des documents officiels (juridiques) pour les citoyens. En Russie, comme dans de nombreux autres pays, le langage juridique, que les linguistes refusent souvent de reconnaître comme jargon professionnel, insistant sur le fait qu'il ne s'agit que d'un style fonctionnel de langage littéraire, s'est beaucoup compliqué au cours de la dernière décennie, notamment dans les constructions syntaxiques utilisées. De ce fait, même les juristes professionnels ont du mal à comprendre les textes des lois, des règlements et de certaines décisions de justice. Les linguistes ont déjà commencé à travailler sur des recettes visant à combattre la complication excessive du langage juridique (un de ces projets est mis en œuvre à l'Institut de recherche sur les questions de langue officielle de l'État, de l'Université d'État de Saint-Pétersbourg, avec le soutien de la Fondation scientifique de Russie), mais pour que ces recettes soient appliquées avec succès dans la pratique, il est nécessaire de reconnaître dans la réglementation juridique au moins le principe essentiel : que tout texte juridique soit compréhensible pour les citoyens auxquels il est adressé. Dans le cas des lois, il doit s'agir du large éventail de ceux dont elles affectent les droits et obligations et qui peuvent être tenus responsables de leur non-respect. Malheureusement, tous les juristes ne reconnaissent pas aujourd'hui de telles exigences, certains insistent sur le fait que les textes juridiques ne doivent être compris que par des professionnels. Cependant, les principes d'un État démocratique ne nous permettent pas de considérer cette dernière approche comme acceptable et l'une des principales exigences pour le développement de la réglementation juridique de la langue en Russie est d'entériner au niveau du principe juridique l'effort obligatoire de clarté générale de tout acte juridique. 